\section{$\underset{\substack{\text { hommes } \\ \text { \& migrations }}}{ }$}

\section{Hommes \& migrations}

Revue française de référence sur les dynamiques

migratoires

1305 | 2014

L'exil chilien en France

\title{
D’Indochine en Lot et Garonne, le périple des métis
}

\section{Dominique Rolland}

\section{(2) OpenEdition \\ Journals}

\section{Édition électronique}

URL : http://journals.openedition.org/hommesmigrations/2759

DOI : 10.4000/hommesmigrations.2759

ISSN : 2262-3353

\section{Éditeur}

Musée national de l'histoire de l'immigration

\section{Édition imprimée}

Date de publication : 1 janvier 2014

Pagination : 160-161

ISBN : 978-2919-040261

ISSN : $1142-852 X$

\section{Référence électronique}

Dominique Rolland, « D'Indochine en Lot et Garonne, le périple des métis », Hommes \& migrations [En ligne], 1305 | 2014, mis en ligne le 10 juillet 2014, consulté le 22 septembre 2020. URL : http:// journals.openedition.org/hommesmigrations/2759; DOI : https://doi.org/10.4000/ hommesmigrations.2759 


\title{
REPÉRAGE
}

\section{D'INDOCHINE \\ EN LOT ET GARONNE LE PÉRIPLE DES MÉTIS}

\begin{abstract}
DOMINIQUE ROLLAND, anthropologue, maître de conférences à l'Institut national des langues et civilisations orientales (Inalco), auteure de Petits Vietnams, histoire des camps de rapatriés d'Indochine (Elytis, 2010).
\end{abstract}

$\grave{A}^{\prime}$ Sainte-Livrade-sur-Lot, le Cafi, c'est bien fini. Les derniers bâtiments sont en cours de destruction tandis que les anciens résidents s'habituent à leurs nouveaux logements, des maisons confortables auxquelles les architectes ont même réussi à donner, avec leurs toits de tuiles, un petit air asiatique. Le panneau de bois délavé qui, depuis cinq décennies, indiquait à l'entrée du camp "Centre d'accueil des Français d'Indochine", autrement dit Cafi, a été enlevé. Enlevé mais néanmoins conservé pour un futur lieu de mémoire qui, un jour peut-être, sera créé. Nul parmi eux n'a en tout cas oublié l'arrivée ici, il y a cinquante-sept ans, après un long voyage en mer de Saigon jusqu'à Marseille et une traversée de la France en bus. Ni même l'immense désillusion qui a suivi... Le Cafi, c'était, il y a quelques mois encore, les longs bâtiments d'une ancienne poudrerie sommairement aménagés en logements pour y héberger des familles rapatriées d'Indochine en 1956. L'hébergement devait être provisoire, il a duré jusqu'à ce qu'un récent programme de requalification et d'aménagement mette un terme à un demi-siècle de précarité et d'oubli. Que s'est-il donc passé ?

\section{Rapatriés en métropole}

En 1956, quelques milliers de Français d'Indochine sont rapatriés en métropole en application des accords de Genève. Parmi eux, une majorité de femmes vietnamiennes seules avec leurs enfants métis, nés de leur union avec un militaire ou un colon français, mais aussi quelques couples, parfois eurasiens, et des vietnamiens de nationalité française. Une partie de ces rapatriés, notamment les plus jeunes et ceux qui avaient déjà un métier, purent rapidement trouver un emploi et quitter les camps d'hébergement. Pour les autres, femmes vietnamiennes isolées et mères de nombreux enfants, couples trop âgés ou malades incapables d'assurer l'existence de leur famille, l'hébergement provisoire prit une allure de long cours. Dans les années 1960, le camp, comme on prit I'habitude de dire, comptait 1200 habitants, dont 700 enfants de moins de 15 ans. Situé à quelques kilomètres de la ville de Sainte-Livrade, il était composé d'une quarantaine de barres disposées autour d'un terrain central. Les logements comprenaient un point d'eau avec un évier dans la pièce commune - mais pas de toilettes, ni de salle de bains -, une cuisinière et un poêle à charbon. Le nombre de chambres variait selon la composition familiale, et les enfants s'y entassaient, souvent dans le même lit.

\section{Relégués de la société française}

La direction du camp était militaire : elle était assurée par des officiers ayant servi en Indochine, censés y avoir acquis de l'expérience, tout comme le médecin ou le prêtre, un missionnaire venu de 
Saigon. La discipline aussi était militaire, avec lever du drapeau et obligation de déclarer toute visite de personne extérieure. Le camp comprenait également un dispensaire, une école et même, pendant quelque temps, une usine de chaussures. Les contacts avec l'extérieur se limitaient au marché du vendredi, où les femmes allaient s'approvisionner. Les paysans des environs prirent cependant l'habitude de venir chercher au camp, pour les récoltes de fruits et de légumes, cette maind'œuvre féminine, démunie, silencieuse et dure à la tâche, ignorante de ses droits et qu'on pouvait sous-payer. Dès qu'ils furent en âge de trouver un emploi mieux rémunéré ou, pour les plus chanceux, de poursuivre leurs études, les adolescents partirent. Les enfants arrivés très jeunes ou nés au camp grandirent quant à eux dans une situation de relégation et de rupture avec la société française, jusqu'à ce qu'à leur tour ils puissent prendre leur autonomie.

Progressivement, cette deuxième génération quitta le camp, y laissant les mères, les frères plus jeunes et quelques-uns de ceux qui jamais ne purent s'intégrer. Ceux qui avaient trouvé un emploi dans la région, ou plus loin, revenaient régulièrement au camp lors de congés et veillaient à procurer à leur mère de quoi améliorer leurs conditions de vie. Le camp pris alors des allures de "petit Vietnam" où la vie s'organisait dans la débrouillardise et la solidarité. Un espace identitaire fort qui devint pour ces déracinés l'unique point d'ancrage.

\section{Raillés, méprisés, insultés}

Pourquoi a-t-on abandonné à leur sort des centaines d'Eurasiens, citoyens français ? En Indochine, la question métisse était très présente, occupant les conversations, faisant l'objet de débats et de controverses, de rapports, de romans d'articles de journaux. La tonalité de ces dires et de ces écrits était le plus souvent péjorative. Les métis étaient raillés, méprisés, souvent insultés, toujours tenus en suspicion. On parlait couramment de la "salle race de métis", on disait d'eux qu' "ils héritaient de toutes les tares", du côté français comme du côté annamite. Les Vietnamiens, quant à eux, à mesure que le nationalisme et les aspirations à l'indépendance grandissaient, ne voyaient plus qu'en eux des larbins de l'ordre colonial et des traîtres à leurs origines. À Saigon, Hanoi, Hué, Tourane, existait une mixophobie' tenace, que l'histoire et les mémoires ont fini par oublier. Il y a peu de temps encore, cette réalité était ignorée ou tenue pour négligeable, l'image devenue valorisante du métissage, promesse de richesse et d'ouverture sur le monde, empêchant que l'on s'interroge sur cette face cachée. ${ }^{2}$

Dans les deux protectorats (Annam, Tonkin) et la colonie de Cochinchine, le concubinage fut une pratique extrêmement fréquente dès la fin du XIXe siècle. L'arrivée nombreuse de militaires, puis de colons, de commerçants et de petits fonctionnaires, majoritairement des hommes jeunes et célibataires, explique ce phénomène. Les militaires ne restent que quelques années et se mettent en ménage le temps de leur séjour. L’armée se montre favorable à ces concubinages qui garantissent au soldat une relative stabilité matérielle et affective. Le problème, ce sont les enfants : bien évidemment, ils ne sont pas reconnus par ces hommes jeunes, dont l'avenir amoureux, familial, professionnel se trouve en France et pour qui l'Indochine n'est qu'une parenthèse. Leur départ laissera donc sur place des femmes avec des enfants en bas âge, soudainement sans ressources. Certaines, faute d'une autre solution, se remettront en ménage avec un nouvel arrivé, dont elles auront d'autres enfants, mais qui à leur tour les abandonnera, les conduisant à une déchéance certaine.

\footnotetext{
1. Le terme est utilisé par Pierre-André Taguieff dans son ouvrage La Couleur et le Sang. Doctrines racistes à la française, Mille et une Nuits, 2002. 2. Le premier ouvrage conséquent sur la question fut la thèse d'Emmanuelle Saada, publiée sous le titre Les Enfants de la colonie. Les métis de l'empire français, entre sujétion et citoyenneté, La Découverte, 2007.
} 


\section{REPÉRAGE}

\section{Indigènes}

Fils de militaires repartis ou morts au combat, enfants de colons de la brousse, rejetons de relations que des fonctionnaires, parfois mariés, entretiennent avec des maîtresses plus ou moins clandestines, les métis abandonnés, de plus en plus nombreux, population de sang-mêlé, posent rapidement problème à la société coloniale et à l'administration.

Un problème juridique d'abord. Dans une société où il n'y a pas de statut intermédiaire entre indigènes et citoyens français, l'enfant métis non reconnu ne peut être qu'indigène. De cette contrainte, découle une interrogation morale : comment un enfant de sang français peut-il être indigène et se voir donc limité dans ses droits ? Le débat porte aussi sur la question de l'acquis et de l'inné : on s'interroge pour savoir s'il ne convient pas de retirer au plus tôt ces enfants du milieu annamite, en misant sur une éducation à la française, qui compenserait les influences néfastes de leur origine maternelle. Un problème social, ensuite. Quand les premières générations arrivent à l'adolescence, ils ne peuvent prétendre qu'aux établissements scolaires franco-indigènes et aux emplois subalternes de l'administration. Comment dès lors éviter d'en faire des déclassés et des aigris ? Se profile également un risque politique : déçus par la France, mus par le ressentiment ou un désir de revanche, ne risquentils pas de devenir des leaders d'une contestation anticoloniale ? Cette crainte se révéla par la suite sans fondement : dans leur immense majorité, les métis répondirent plutôt à l'injonction qui leur était faite de se conformer au modèle dominant,età l'imagedupère.Ces préoccupations sont si présentes que, dès la fin du XIXe siècle, apparaissent des sociétés de défense de l'enfance métisse abandonnée, en 1896 à Saigon, en 1898 à Hanoi. Ces associations regroupent des fonctionnaires, des notables et quelques colons émus de ces abandons et révoltés par les préjugés dont les métis font l'objet. Elles fournissent une aide financière aux mères les plus démunies ou placent les enfants dans les institutions: pour leur bien, il faut soustraire ces enfants à l'influence néfaste du milieu vietnamien et les éduquer "à la française". Certains colons ou fonctionnaires vont ainsi jusqu'à confier d'eux-mêmes leurs enfants métis à des institutions religieuses ou à les envoyer en France auprès d'un parent qui accepte de s'en charger ${ }^{3}$. Le contact avec la mère vietnamienne et sa famille est alors définitivement, et volontairement, rompu, dans le souci d'intégrer le jeune métis à la société française.

\section{Double culture}

En 1928, un décret ${ }^{4}$ règle en partie les problèmes juridiques en facilitant l'accès à la citoyenneté française pour les métis non reconnus : il suffit d'un témoignage ou d'un certificat médical attestant une origine européenne. Cela ne met pas fin aux préjugés, mais permet à de nombreux métis d'obtenir, dans les différentes administrations, des postes de responsabilité et même d'intégrer l'armée au rang d'officier. Nombre de métis sont recrutés dans la police, les douanes, la sûreté, les prisons, à des fonctions de surveillance, d'enquête, d'interrogatoires, où leur double culture, leur connaissance de la langue et des réseaux, sont particulièrement appréciées.

\footnotetext{
3. En 1931, une de ces associations organisa même le transfert en Normandie, dans la région de Coutances, d'une centaine d'enfants et d'adolescents placés dans des familles ou dans des internats. 4. Décision de la cour d'appel de Hanoi en 1926, puis décret du 8 novembre 1928 : "Tout individu né sur le territoire de l'Indochine, de parents dont l'un, demeuré inconnu, est présumé de race française, peut obtenir la reconnaissance de la qualité de Français."
} 
Mais cela ne fait que renforcer le ressentiment vietnamien à leur égard. Un ressentiment qui s'accroît à mesure que le nationalisme et la contestation du système colonial gagnent en puissance, et qui finit après 1954, par rendre inéluctable le départ : toute la population métisse du Nord-Vietnam ne voit plus que son salut dans la métropole, sans imaginer un instant l'immense désillusion qui va suivre.

$\mathrm{Au} \mathrm{Cafi}$, on retrouve toutes les expressions possibles du métissage. Car à partir de 1945, arrivent en Indochine les unités combattantes du Corps expéditionnaire français en Extrême-Orient (CEFEO), qui vont intervenir sur le terrain jusqu'à la défaite de Dîen Bîen Phu en 1954, et maintenir une présence au sud jusqu'en 1956. Or, recruté dans toute I'Union française, ce corps d'armée, qui représentera jusqu'à 180000 hommes, compte des Sénégalais, des Algériens, des Marocains, des Tunisiens, des Congolais, des Réunionnais, des Canaques, des Martiniquais, des Guadeloupéens, des Guyanais, des Malgaches, des Comoriens, mais aussi des légionnaires espagnols, polonais, russes, allemands, italiens, roumains, bulgares, allemands. Des légionnaires et des soldats coloniaux qui prennent eux aussi l'habitude de se mettre en ménage avec des femmes annamites.

Aujourd'hui encore, les personnes extérieures au camp sont souvent surprises de découvrir ces métis aux physiques si divers, partageant une seule langue, le vietnamien, et se revendiquant de leurs seules origines vietnamiennes. Élevés par des mères qui ne parlaient pas français et n'avaient que peu de contacts avec la société française, sans illusions sur l'attitude de la France à leur égard, c'est cette seule identité première qui compte pour eux. "Être du camp", avoir grandi dans cette minuscule enclave de culture vietnamienne, où toutes les mères étaient des mères, et tous les enfants des frères, est le point d'ancrage qui les rassemble.

\section{Contraintes contradictoires, choix impossibles}

L'identité métisse s'est forgée en Indochine sous l'emprise de contraintes contradictoires et de choix impossibles : pour être reconnu par la société coloniale, il fallait être assimilé au plus près, dans le langage, dans l'habillement, dans les goûts, dans l'attitude, et gommer la part vietnamienne de soi.

Dès le début, la double identité des métis a été marquée par la question d'une fidélité impossible, car, quoi qu'ils fassent, être fidèle à une part d'eux-mêmes impliquait toujours, nécessairement, être traître à l'autre, sans que cette tension ne se relâche jamais.

Au contraire, elle n'a fait que s'accentuer avec les luttes anticoloniales, la guerre, le rapatriement en France. Rien de surprenant à ce que la difficulté à se saisir, à s'affirmer, le doute permanent sur soi, la culpabilité diffuse taraudent encore aujourd'hui ces métis.

La France, exigeant une allégeance sans faille, a engendré d'intimes et douloureuses déchirures avec le Vietnam, pour finalement condamner ces métis à l'oubli dans ces baraquements précaires, auxquels ils ont su donner une âme.

Voilà pourquoi la préservation de la mémoire est si importante aujourd'hui. Les traces matérielles du Cafi ont été déblayées par les bulldozers.

La première génération doucement s'efface : les vieilles dames en chapeau conique bavardant sur le seuil des maisons se font de plus en plus rares. Mais la deuxième génération, abordant la soixantaine, craint que son histoire ne sombre dans l'oubli. "Tout ce que nos parents ont enduré, tout ce que nous avons souffert, ne serait rien?" demandent-ils, inquiets. Les plus jeunes haussent les épaules, désabusés : la reconnaissance, ils n’y croient plus. 